Authors' Contribution: A Study Design B Data Collection C Statistical Analysis D Data Interpretation E Manuscript Preparation $\mathbf{F}$ Literature Search G Funds Collection

\section{An examination of the hamstring and the quadriceps muscle kinematics during the front and back squat in males}

\author{
Jonathan Sinclair ABCDEF, Darrell Brooks ABCDEF, Stephen Atkins ABCDEF \\ Division of Sport Exercise and Nutritional Sciences, School of Sport \& Wellbeing, College \\ of Health \& Wellbeing, University of Central Lancashire, United Kingdom
}

\section{abstract}

Background

Material/Methods

Results

Conclusions

Key words
The aim of the current investigation was to examine the influence of the front and back squat variants on the hamstring and the quadriceps muscles kinematics.

Eighteen male participants were recruited with 1 repetition maximum values of 122.7 \pm 16.4 and $88.7 \pm 13.9 \mathrm{~kg}$ for the back and front squat lifts. Participants completed both back and front squats at $70 \%$ of their front squat 1 repetition maximum. The hamstring and the quadriceps muscle kinematics were determined during kinetic situations using motion capture data, in addition to segmental and joint kinematics.

Differences between squat conditions were examined using paired samples t-tests. The results showed that there were no differences in either segmental/joint or muscle kinematics between the front and back squat lifts.

These findings lead to the conclusion that neither the front nor the back squat conditions differ in terms of their eccentric and concentric recruitment of the quadriceps and hamstring muscles.

squat, hamstring, quadriceps, kinematics, musculoskeletal

\section{article details}

Article statistics

Full-text PDF:

Copyright

Indexation:

Funding:

Conflict of interest: Corresponding author:

Open Access License:
Word count: 2,737; Tables: 3; Figures: 3; References: 18

Received: October 2015; Accepted: December 2016; Published: March 2017

http://www.balticsportscience.com

(c) Gdansk University of Physical Education and Sport, Poland

AGRO, Celdes, CNKI Scholar (China National Knowledge Infrastructure), CNPIEC, De Gruyter - IBR (International Bibliography of Reviews of Scholarly Literature in the Humanities and Social Sciences), De Gruyter - IBZ (International Bibliography of Periodical Literature in the Humanities and Social Sciences), DOAJ, EBSCO - Central $\&$ Eastern European Academic Source, EBSCO - SPORTDiscus, EBSCO Discovery Service, Google Scholar, Index Copernicus, J-Gate, Naviga (Softweco, Primo Central (ExLibris), ProQuest - Family Health, ProQuest - Health \& Medical Complete, ProQuest - Illustrata: Health Sciences, ProQuest - Nursing \& Allied Health Source, Summon (Serials Solutions/ProQuest, TDOne (TDNet), Ulrich's Periodicals Directory/ulrichsweb, WorldCat (OCLC)

\section{sectors.}

Authors have declared that no competing interest exists.

Dr. Jonathan Sinclair; Division of Sport, Exercise and Nutritional Sciences, School of Sport \& Wellbeing; University of Central Lancashire, Preston, Lancashire PR1 2HE; e-mail: jksinclair@uclan.ac.uk

This is an open access article distributed under the terms of the Creative Commons Attribution-Non-commercial 4.0 International (http://creativecommons.org/licenses/by-nc/4.0/), which permits use, distribution, and reproduction in any medium, provided the original work is properly cited, the use is non-commercial and is otherwise in compliance with the license. 


\section{INTRODUCTION}

The barbell squat is one of the most frequently used and fundamental exercises in the field of strength and conditioning [1]. The objective of the squat is primarily to recruit and strengthen the musculature associated with the hip and knee joints [2]. Importantly, the squat is known to exhibit biomechanical similarities with a wide range of sports movements and thus is included in most training routines with the goal of enhancing athletic performance [3]. The squat itself has two principal variants: the back and the front squat lifts.

A considerable amount of research has been conducted by both sports scientists and biomechanists regarding the mechanics of the squat; however, there is comparatively little information available regarding the differences between the front and back squat variants. Russell \& Phillips [4] investigated the influence of the front and back squat on the sagittal plane kinematics and joint torques. They showed that no differences in joint torques were evident between squat conditions, but the back squat was associated with greater flexion of the trunk segment. Diggin et al. [5] investigated differences in the lower limb and trunk kinematics between the front and the back squat. Their findings concur with those of Russell \& Phillips [4] in that performing the back squat was associated with significantly greater trunk flexion in comparison to the front squat.

Gullett et al. [6] comparatively examined the effects of performing the front and back squat variants on knee joint forces and muscle activation of the quadriceps, hamstring and erector spinae. Their observations confirmed that the back squat was associated with greater knee forces compared to the front squat, but no differences in muscle activation were shown. Stuart et al. [7] also examined the effects of the two squat variants on knee joint kinetics and muscle activation. They showed that neither knee joint forces nor muscle activation differed as a function of different squat techniques. Sinclair et al. [8] investigated the differences in the patellofemoral joint kinetics between the front and back squat. Their findings revealed that the back squat was associated with significant increases in both patellofemoral force and pressure in comparison to the front squat, which they proposed may be associated with an increased risk from knee pathology. Finally, Sinclair et al. [9] examined the influence of the front and back squat lifts on the loads experienced by the Achilles tendon. The results of this investigation showed that the peak loads experienced by the Achilles tendon were significantly larger during the back squat compared to the front squat.

Whilst there is some literature which has investigated differences between the front and the back squat, there has yet to be a comparative examination of the muscle kinematics between the two squatting modalities. A lack of suitable measurement techniques capable of quantifying muscle mechanics is a key limitation; however, specific software now exists which is able to provide dynamic simulations of skeletal muscle kinematics during dynamic situations [10].

The aim of the current investigation, therefore, was to examine the influence of the front and the back squat variants on the hamstring and the quadriceps muscles kinematics. A study of this nature may provide important information for those who seek to understand skeletal muscle control and for athletes who habitually utilize the squat in their resistance training. 


\section{MATERIAL AND METHODS}

\section{PARTICIPANTS}

Eighteen male participants (age 25.9 SD 5.1 years, height 1.74 SD $0.12 \mathrm{~m}$ and body mass 77.44 SD $5.29 \mathrm{~kg}$ ) volunteered to take part in the current investigation. Participants had $6.55 \pm 2.11$ years of experience in squat lifting with 1 repetition maximum values of $122.7 \pm 16.4$ and $88.7 \pm 13.9 \mathrm{~kg}$ for the back and front squat lifts, respectively. Participants trained at least 3 times per week and habitually utilized both squatting techniques as part of their resistance training routine. Ethical approval was obtained from the University Ethics Committee, and the procedures outlined in the Declaration of Helsinki were followed.

\section{PROCEDURE}

Participants completed five repetitions in each squat condition, using their normal back and front squat technique. The load was consistent for both conditions, with participants lifting $70 \%$ of their front squat 1 repetition maximum. Participants completed their squats in a randomised order to control for any order effects.

Kinematic information was captured at $250 \mathrm{~Hz}$ using an eight camera optoelectric motion analysis system (QualisysTM Medical AB, Gothenburg, Sweden). To define the anatomical frames of the trunk, pelvis, thighs, shanks and feet retroreflective markers were placed at the C7, T12 and xiphoid process landmarks and also positioned bilaterally onto the acromion process, iliac crest, anterior superior iliac spine, posterior superior iliac spine, medial and lateral malleoli, medial and lateral femoral epicondyles and greater trochanter. Carbon-fibre tracking clusters comprising of four non-linear retroreflective markers were positioned bilaterally onto the thigh and shank segments. Static calibration trials were obtained with the participant in the anatomical position in order for the positions of the anatomical markers to be referenced in relation to the tracking clusters/markers.

\section{DATA PROCESSING}

Marker trajectories were filtered $6 \mathrm{~Hz}$ using a low pass Butterworth 4 th order zero-lag filter and analysed using Visual 3D (C-Motion, Germantown, MD, USA). All temporal information was normalized to $100 \%$ of the squat movement. The timing of the initiation and termination of the squat movement for both techniques were taken as the instances of maximum hip extension in accordance with those of Sinclair et al. [11]. For the current study segmental kinematics of the thorax and pelvic segments were examined in addition to joint kinematics of the hip and knee.

OpenSim software was used to quantify muscle-tendon lengths during the kicking movements [10]. Muscle kinematics were quantified using the gait2392 model using Opensim v3.2. This model corresponds to the eight segments exported from Visual 3D and features ninety-two muscles, eighty-six of which are centred around the lower extremities and six are associated with the pelvis and the trunk. The muscle properties were modelled using the Hill recommendations based on the associations between force-velocity-length [12]. These muscle properties were then scaled based on each participant's height and body mass based on the recommendations of Delp et al. [13]. 
Muscle-tendon lengths are determined by the positions of their proximal and distal muscle origins. The muscle-tendon units which were evaluated as part of the current research were the rectus femoris, vastus medialis, vastus lateralis, vastus intermedius, biceps femoris long head (LH), biceps femoris short head (SH), semimembranosus and semitendinosus. All muscle-tendon units were normalized to their length during the static calibrations trials. Muscle kinematic parameters that were extracted for statistical analysis were: 1) the peak length during the squat movement 2) the eccentric strain (representative of the maximum increase in length divided by standing length, 3 ) the concentric strain (representative of the maximum decrease in length divided by the standing length. All values were normalized to resting muscle length as determined via the static trial.

\section{STATISTICAL ANALYSES}

Differences between muscles and the two squat conditions were examined using 4 (muscle) x 2 (squat condition) repeated measures ANOVA's for each muscle group. Significance was accepted at the $p<0.05$ level [14]. Significant interactions were further investigated using simple main effects. Effect sizes were quantified using partial eta ${ }^{2}\left(\mathrm{p \eta}^{2}\right)$. The Shapiro-Wilk statistic for each condition confirmed that the data were normally distributed. Finally, the similarity of the muscle/joint kinematics waveforms between squat conditions were examined using intraclass correlations (ICC). All statistical procedures were conducted using SPSS v22.0 (SPSS Inc., Chicago, IL, USA).

\section{RESULTS}

Figures 1-3 and Tables 1-3 present joint and muscle kinematics during the squat. The results indicate that there were no differences in joint/muscles kinematics between front and back squat techniques and that a high level of similarity was evident between waveforms.
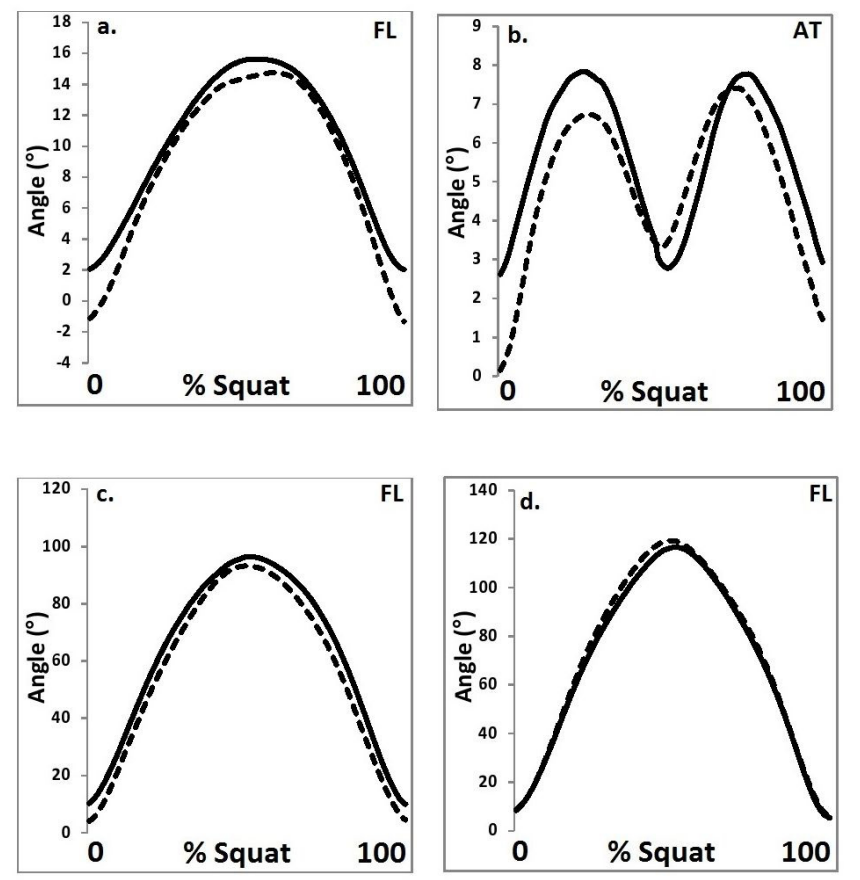

Fig. 1. Kinematics as a function of the front and the back squat lifts $a .=$ trunk flexion, $b .=$ pelvic tilt, c. = hip angle, d. = knee angle (black = back \& dash $=$ front) $(A T=$ anterior tilt, FL = flexion) 

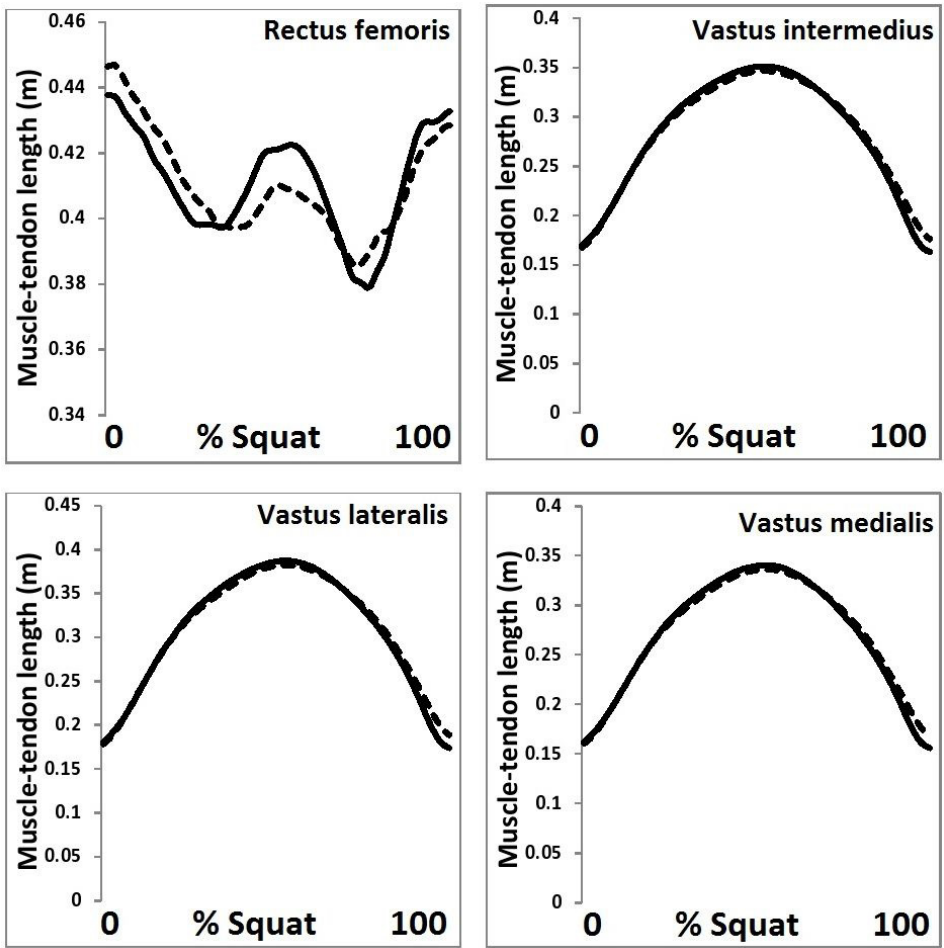

Fig. 2: Quadriceps kinematics as a function of front and back squat lifts (black $=$ back $\&$ dash $=$ front)
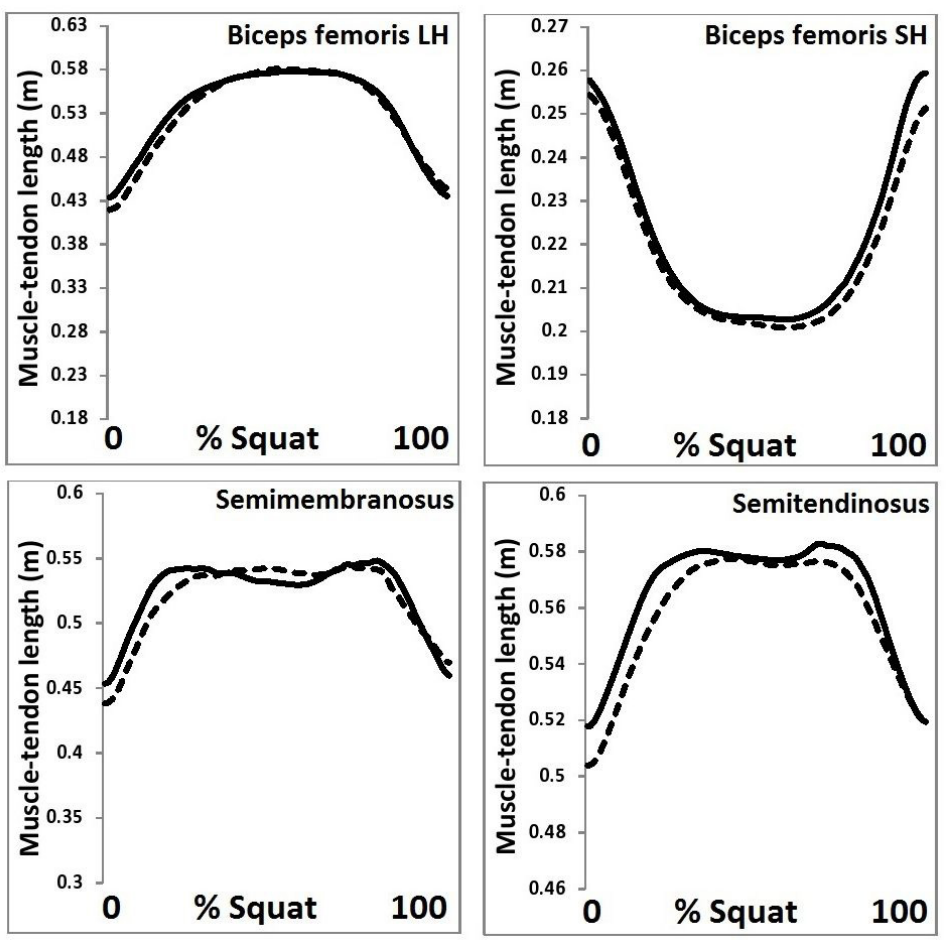

Fig. 3: Hamstring kinematics as a function of front and back squat lifts (black $=$ back $\&$ dash $=$ front) 
Table 1. Quadriceps kinematics (Mean $\pm \mathrm{SD}$ ) as a function of both lifts

\begin{tabular}{|c|c|c|c|c|}
\hline & \multicolumn{2}{|c|}{ Back } & \multicolumn{2}{|c|}{ Front } \\
\hline & Mean & SD & Mean & SD \\
\hline Rectus femoris peak length (\%) & 83.84 & 12.16 & 82.69 & 14.93 \\
\hline Rectus femoris eccentric strain (\%) & 16.16 & 12.14 & 19.34 & 11.50 \\
\hline Rectus femoris concentric strain (\%) & 15.28 & 10.80 & 15.26 & 9.28 \\
\hline Vastus intermedius peak length (\%) & 209.37 & 16.72 & 207.63 & 21.17 \\
\hline Vastus intermedius eccentric strain (\%) & 109.37 & 16.27 & 108.05 & 17.01 \\
\hline Vastus intermedius concentric strain (\%) & 112.33 & 15.69 & 103.79 & 24.62 \\
\hline Vastus lateralis peak length (\%) & 216.73 & 21.93 & 215.19 & 23.61 \\
\hline Vastus lateralis eccentric strain (\%) & 116.73 & 18.69 & 115.56 & 19.02 \\
\hline Vastus lateralis concentric strain (\%) & 119.49 & 17.20 & 110.88 & 27.14 \\
\hline Vastus medialis peak length (\%) & 212.10 & 17.54 & 210.61 & 22.32 \\
\hline Vastus medialis eccentric strain (\%) & 112.12 & 17.09 & 111.05 & 17.96 \\
\hline Vastus medialis concentric strain (\%) & 115.11 & 16.28 & 106.95 & 25.52 \\
\hline
\end{tabular}

Table 2. Hamstring kinematics (Mean \pm SD) as a function of both lifts

\begin{tabular}{|c|c|c|c|c|}
\hline & \multicolumn{2}{|c|}{ Back } & \multicolumn{2}{|c|}{ Front } \\
\hline & Mean & SD & Mean & SD \\
\hline Biceps femoris LH peak length (\%) & 134.58 & 10.23 & 135.90 & 10.75 \\
\hline Biceps femoris LH eccentric strain (\%) & 34.58 & 10.23 & 38.97 & 9.92 \\
\hline Biceps femoris LH concentric strain (\%) & 34.39 & 10.74 & 33.46 & 13.57 \\
\hline Biceps femoris SH peak length (\%) & 78.42 & 2.83 & 77.62 & 5.06 \\
\hline Biceps femoris SH eccentric strain (\%) & 21.58 & 2.83 & 20.94 & 2.58 \\
\hline Biceps femoris SH concentric strain (\%) & 22.20 & 3.36 & 19.84 & 3.15 \\
\hline Semimembranosus peak length (\%) & 126.27 & 14.14 & 126.11 & 14.93 \\
\hline Semimembranosus eccentric strain (\%) & 26.27 & 14.14 & 28.75 & 11.01 \\
\hline Semimembranosus concentric strain (\%) & 24.83 & 13.51 & 21.77 & 12.83 \\
\hline Semitendinosus peak length (\%) & 113.96 & 6.02 & 113.13 & 6.51 \\
\hline Semitendinosus eccentric strain (\%) & 13.96 & 6.02 & 15.83 & 4.94 \\
\hline Semitendinosus concentric strain (\%) & 13.67 & 5.99 & 12.85 & 5.86 \\
\hline
\end{tabular}

\section{SEGMENT/JOINT KINEMATICS}

No significant $(p>0.05)$ differences between segment/joint kinematics were observed between squat conditions (Fig. 1).

\section{QUADRICEPS KINEMATICS}

For the peak muscle length a significant main effect $\left(p<0.05, \mathrm{p}^{2}=0.40\right.$ ) was shown for the muscle. Post-hoc analysis showed that the peak length of the rectus femoris was significantly shorter in comparison to the vastus lateralis, vastus medialis and vastus intermedius. For the maximum eccentric strain a significant main effect $\left(p<0.05, \mathrm{p}^{2}=0.68\right)$ was shown for the muscle. Post-hoc analysis showed that the maximum eccentric strain of the rectus femoris was significantly reduced in comparison to the vastus lateralis, vastus medialis and vastus intermedius. For the maximum concentric strain a significant main effect $\left(p<0.05, \mathrm{p \eta}^{2}=0.67\right.$ ) was shown for the muscle. Post-hoc analysis showed that the maximum concentric strain of the rectus femoris was significantly reduced in comparison to the vastus lateralis, vastus medialis and vastus intermedius (Fig. 2; Table 1). 


\section{HAMSTRING KINEMATICS}

For the peak muscle length a significant main effect $\left(p<0.05, \mathrm{p}^{2}=0.35\right)$ was shown for the muscle. Post-hoc analysis showed that the peak length of the biceps femoris $\mathrm{SH}$ was significantly shorter in comparison to the biceps femoris LH, semimembranosus and semitendinosus (Fig. 3; Table 2).

\section{WAVEFORM SIMILARITY}

Table 3. Similarity (ICC) between lifts

\begin{tabular}{cl}
\hline Rectus femoris & 0.993 \\
\hline Vastus intermedius & 0.997 \\
\hline Vastus lateralis & 0.998 \\
\hline Vastus medialis & 0.998 \\
\hline Biceps femoris LH & 0.998 \\
\hline Biceps femoris SH & 0.981 \\
\hline Semimembranosus & 0.995 \\
\hline Semitendinosus & 0.997 \\
\hline Trunk & 0.983 \\
\hline Pelvic tilt & 0.919 \\
\hline Hip & 0.992 \\
\hline Knee & 0.999 \\
\hline
\end{tabular}

High levels of similarity (ICC $\geq 0.919$ ) were evident between waveforms for the two squat conditions.

\section{DISCUSSION}

The aim of the current investigation was to determine the effects of the front and back squat lifts on the hamstring and the quadriceps muscle kinematics. To the authors' knowledge this represents the first comparative investigation to examine differences in muscle kinematics during the front and the back squat lifts.

The first key observation was that no differences in either the hamstring or the quadriceps muscle kinematics were observed between the front and the back squat conditions. This concurs with the kinematic analyses which also showed that no differences were evident between squat conditions. That no differences in kinematics were evident between conditions opposes the observations of Russell \& Phillips [4] and Diggin et al. [5], who showed increased trunk flexion in the back squat condition.It is proposed that this divergence in findings may relate to differences in the measurement technique between the studies. Both Russell \& Phillips [4] and Diggin et al. [5] utilized a 2D procedure to quantify trunk flexion, which is in contrast to the current investigation, whereby a 3D six degrees of freedom approach was employed. The key implication from these findings in relation to muscle kinematics is that the hamstring and the quadriceps muscle groups exhibit similar magnitudes of eccentric and concentric lengthening and shortening between the two squat modalities.

For the quadriceps muscle group differences were shown for all measurements with the rectus femoris exhibiting differences from the vastus lateralis, vastus medialis and vastus intermedius. During the descent and ascent phase the vastus lateralis, vastus medialis and vastus intermedius muscles all exhibited eccentric lengthening and concentric shortening of similar magnitude relative 
to resting length. This is to be expected as both the hip and the knee joints exhibit flexion during the descent phase of the squat and extension during the ascent phase [11]. As the vastus lateralis, vastus medialis and vastus intermedius attach proximally to the anterio-proximal aspect of the femur and distally into the quadriceps tendon the distance between insertion points will increase during flexion and decrease during extension. Given the phasic pattern of eccentric lengthening and concentric shortening exhibited by these muscles, it is clear that muscle potentiation mediated by the stretch shorten cycle action $[15,16]$ is utilized by these muscles to lift the heavy loads associated with the squat.

A further key observation is that the rectus femoris muscle exhibited a distinct pattern of lengthening and shortening in comparison to the vastus lateralis, vastus medialis and vastus intermedius. It is likely this relates to the distinct proximal insertion point of the rectus femoris at the anterior superior iliac spineas opposed to the anterio-proximal aspect of the femur. Because the pelvic segment tilts anteriorly during the squat this leads to a reduction in the length of the rectus femoris muscle tendon unit, despite the knee joint being in a flexed position. Muscle force potentiation mediated by the stretch shorten cycle is important during the squat where large masses are typically lifted [17]. As the rectus femoris does not experience eccentric lengthening during the descent phase, it can be concluded that the rectus femoris does not store any elastic energy that may be released during the ascent, indicating that this muscle does not contribute optimally to the squat. This supports the notion proposed by Escamilla [2] that the rectus femoris may not be utilized to its greatest potential during the squat and that anterior tilt of the pelvis and trunk segments should be minimized in order to maximize the contribution of the rectus femoris.

In addition to the vastus lateralis, vastus medialis and vastus intermedius muscles the biceps femoris LH, semimembranosus and semitendinosus also exhibited a phasic pattern of eccentric lengthening and concentric shortening of similar magnitude relative to resting length during the descent and ascent phases. This observation is an interesting one in that typically the hamstrings are considered to be antagonistic to the quadriceps [18] serving primarily to provide sagittal plane flexion of the knee joint. It is proposed that this observation is due to the proximal and distal attachment positions of these muscles at the ischial tuberosity and to the proximal end of the tibia/fibula. Because the hip and knee joints exhibit flexion and the pelvis tilts anteriorly during the descent phase of the squat, this means that the linear distance between the ischial tuberosity and the proximal end of the tibia/fibula increases. This indicates that the biceps femoris LH, semimembranosus and semitendinosus may also store eccentric elastic energy in the descent phase that is released during the concentric ascent phase of the lift.

\section{CONCLUSIONS}

In conclusion, whilst the biomechanics of the squat lift have been extensively examined, the current knowledge is limited both with regards to differences between front and back squat lifts and with regards to the kinematics of the hamstring and the quadriceps muscles. The current investigation addresses this by providing a comparative investigation and analysis of the hamstring 
and the quadriceps muscles kinematics during the front and the back squat lifts. The results indicate that no differences exist between the two squat modalities but nonetheless important information regarding the phasic lengthening/shortening of the muscles was documented, which may improve our understanding of how these key muscles function during the squat.

\section{REFERENCES}

[1] Schoenfeld BJ. Squatting kinematics and kinetics and their application to exercise performance. J Strength Condit Res. 2010;24:3497-3506.

[2] Escamilla RF. Knee biomechanics of the dynamic squat exercise. Med Sci Sport Exerc. 2001;33:127141.

[3] Senter C, Hame SL. Biomechanical analysis of tibial torque and knee flexion angle: Implications for understanding knee injury. Sport Med. 2006;36:635-641.

[4] Russell PJ, Phillips SJ. A preliminary comparison of front and back squat exercises. Res Q Exerc Sport. 1989;60:201-208.

[5] Diggin D, O’Regan C, Whelan N, Daly S, McLoughlin V, McNamara L, Reilly A. A biomechanical analysis of front versus back squat: injury implications. Port J Sport Sci. 2011;11:643-646.

[6] Gullett JC, Tillman MD, Gutierrez GM, Chow JW. A biomechanical comparison of back and front squats in healthy trained individuals. J Strength Condit Res. 2009;23:284-292.

[7] Stuart MJ, Meglan DA, Lutz GE, Growney ES, An KN. Comparison of intersegmental tibiofemoral joint forces and muscle activity during various closed kinetic chain exercises. Am J Sports Med. 1996;24:792-799.

[8] Sinclair J, Atkins S, Kudiersky N, Taylor PJ and Vincent H. Effects of front and back squat techniques on patellofemoral joint kinetics in males. J Biomed Eng Inf. 2015;2:1-6.

[9] Sinclair J, Edmundson C, Atkins S, Taylor PJ and Vincent H. Effect of front and back squat techniques on peak loads experienced by the Achilles tendon. Balt J Health Phys Act. 2015;7(3):7-12.

[10] Delp SL, Anderson FC, Arnold AS, et al. OpenSim: open-source software to create and analyze dynamic simulations of movement. IEEE Trans Biomed Eng. 2007;54:1940-1950.

[11] Sinclair, J, McCarthy, D, Bentley, I, Hurst, HT, Atkins, S. The influence of different footwear on 3-D kinematics and muscle activation during the barbell back squat in males. Eur J Sport Sci. 2015;15(7):583-90.

[12] Zajac FE. Muscle and tendon: properties, models, scaling, and application to biomechanics and motor control. Crit Rev Biomed Eng. 1989;17:359-411.

[13] Delp SL, Loan JP, Hoy MG, Zajac FE, Topp EL, Rosen JM. An interactive graphics-based model of the lower extremity to study orthopaedic surgical procedures. IEEE Trans Biomed Eng. 1990;37:757-767.

[14] Sinclair J, Taylor PJ, Hobbs SJ. Alpha level adjustments for multiple dependent variable analyses and their applicability - A review. Int J Sport Sci Eng. 2013;7:17-20.

[15] Cavagna GA, Dusman B, Margaria R. Positive work done by a previously stretched muscle. J Appl Physiol. 1968;24:21-32.

[16] Komi PV. Stretch-shortening cycle: a powerful model to study normal and fatigued muscle. J Biomech. 2000;33:1197-1206.

[17] Manabe, Y, Shimada, K, Ogata, M. Effect of slow movement and stretch-shortening cycle on lower extremity muscle activity and joint moments during squat. J Sport Med Phys Fit. 2007;47:1-12.

[18] Kellis E. Quantification of quadriceps and hamstring antagonist activity. Sports Med. 1998;25:37-62.

Cite this article as:

Sinclair J, Brooks D, Atkins S. An examination of the hamstring and the quadriceps muscle kinematics during the front and back squat in males. Balt J Health Phys Act. 2017;9(1):37-45. 